\title{
Study of Binaural Auditory Cortical Response in Children with History of Recurrent Otitis
}

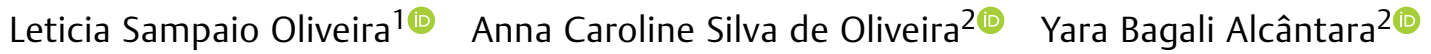 \\ Carolina Almeida Vieira ${ }^{2}$ Dayse Mayara de Oliveira Ferreira ${ }^{3}$ Eduardo Federighi Baisi Chagas ${ }^{4}$ \\ Ana Claudia Figueiredo Frizzo ${ }^{2}$ \\ ${ }^{1}$ Department of Speech Therapy, Faculty of Medicine, Universidade \\ de São Paulo, São Paulo, SP, Brazil \\ 2 Department of Speech Therapy, Universidade Estadual Paulista, \\ Address for correspondence Leticia Sampaio Oliveira, PhD, Av. Higino \\ Marília, SP, Brazil \\ ${ }^{3}$ Department of Speech Therapy, Universidade de São Paulo, Bauru, \\ SP, Brazil \\ ${ }^{4}$ Department of Biostatistics at the Universidade de Marília \\ (UNIMAR), Marília, SP, Brazil \\ Int Arch Otorhinolaryngol 2021;25(4):e490-e495. \\ Muzi Filho, 737 - PORTARIA A - Mirante, Marília - SP, 17525-900, Brazil \\ (e-mail: leticiaoliveira.fono@gmail.com).
}

received

April 8, 2020

accepted

August 5, 2020

published online

November 30, 2020
Introduction Any type of sensory deprivation in childhood resulting from conductive hearing loss may impair the development of peripheral and central auditory pathway structures with negative consequences for binaural processing.

Objective To characterize and compare monoaural and binaural auditory responses in neonates and children without and with a history of recurrent otitis.

Methods The study included participants from 0 to 8 years and 11 months old, in good general health conditions, of both genders, divided into a control group, with no history of otitis, and a study group, with history of recurrent otitis. Cortical potential with speech stimulus /ba/-/da/ was used as collection procedure. The arithmetic calculation of the 512 points of the wave was performed to obtain the grand average of the waves of the subjects in both groups. The Shapiro-Wilk and mixed repeated measures analysis of covariance (ANCOVA) statistical tests were performed to analyze the group effect, the condition, and the interaction (group versus condition) controlling the effect of the age-sex covariable.

Results There was a statistically significant difference between the groups for all latency values; and for the P1, N1, P2, and N2 latencies, the differences between the groups occurred in the three analyzed conditions (right and left ears and binaural), revealing the influence of sensory deprivation. There were no significant differences in relation to wave amplitudes.

Conclusion There are differences in the cortical potential with speech stimuli and in the binaural interaction component of children with and without history of recurrent otitis.

\footnotetext{
November 30.2020
}

DOI https://doi.org/

10.1055/s-0040-1718527. ISSN 1809-9777.

\footnotetext{
(c) 2020. Fundação Otorrinolaringologia. All rights reserved.

This is an open access article published by Thieme under the terms of the Creative Commons Attribution-NonDerivative-NonCommercial-License, permitting copying and reproduction so long as the original work is given appropriate credit. Contents may not be used for commercial purposes, or adapted, remixed, transformed or built upon. (https://creativecommons.org/ licenses/by-nc-nd/4.0/) Thieme Revinter Publicações Ltda., Rua do Matoso 170, Rio de Janeiro, RJ, CEP 20270-135, Brazil
} 


\section{Introduction}

The first 2 years of an individual's life are the most significant for the development of auditory and language skills. Neuroplasticity during this period is at its maximum, and the cortex is more responsive. ${ }^{1}$ Thus, when in contact with external stimuli, the child's auditory system reaches more advanced stages of maturation, according to the age and auditory experience, which favors language acquisition.

Binaurality is the central auditory nervous system's (CANS) ability to receive sound information from both ears (right and left), analyze, integrate, and separate sound information. This binaural processing of information ensures the understanding of different sounds and enables the individual to communicate in environments with different sound sources, difficult listening conditions and allows efficient communication.

Such binaural mechanisms occur from the perception of minimal acoustic differences in the phase, frequency, intensity, and time of a sound, in each ear. ${ }^{2}$ Understanding these acoustic parameters requires the brain to perceive interaural time differences (ITD), for example, to enable specific functions, such as sound localization. ${ }^{3}$

It is noteworthy that such mechanisms and binaural skills, such as understanding speech in noise, will be improved according with the individual's age, maturation, and hearing experience.

The presence of transient hearing loss in childhood causes fluctuation in hearing and sensory deprivation. Consequently, it impairs the development of peripheral and central auditory pathway structures and interferes with these binaural mechanisms. ${ }^{4}$

The most common cause of conductive hearing loss in children is related to otitis media, defined as an inflammation of the middle ear, often associated with fluid increase in this region, which may or may not be infected by viruses or bacteria..$^{5}$ Otitis media manifestation is silent, as it may be associated with infections, inflammations, or extrinsic factors, such as adenoids. ${ }^{6}$

Among the different audiological procedures that assess the integrity of the CANS, we highlight auditory evoked potentials. Cortical auditory evoked potential (CAEP) is a diagnostic test that assesses the electrophysiological response and enables the analysis of the electrical responses generated in the auditory pathway with monoaural and binaural stimulation ${ }^{7}$; it can be used to study the binaural interaction component (BIC).

The binaural interaction component (BIC) is obtained through CAEP responses and is derived by the algebraic sum of the monoaural responses, right and left, subtracted from the response obtained with bilateral stimulation. For this reason, it reflects the response of the electrical activity of specifically binaural neurons and is very useful to investigate structures of the CANS and the functioning of the binaural auditory abilities of sound localization, for example. ${ }^{8}$

One study performed BIC on subjects with normal hearing using the rarefaction click stimulus, and the results demonstrated that BIC with long-latency potentials consisted of the appearance of two negative peaks and one positive peak. The author described strong evidence that binaural processing in the auditory pathway may bring important information regarding the activity in the thalamocortical portions of the auditory pathways and the auditory skills linked to linguistic auditory recognition. ${ }^{8}$

Studies that directly link BIC with sensory deprivation resulting from middle ear changes are unprecedented in the literature. In this area, a more recent study was found regarding the results of auditory evoked potentials (AEPs) in a child population with otitis media, although not related to BIC. Such studies demonstrated that CAEP in children with otitis media may have increased latency, latency asymmetries between the right ear (RE) and left ear (LE), and difficulties in binaural interaction. ${ }^{9}$ Binaural interaction component is believed to bring important information in addition to these findings in the literature on binaural auditory processing, which reflects the innovation of this study for the scientific community.

The results of the present study may help to direct clinical intervention and promote new preventive or treatment approaches aimed at binaural stimulation in children with history of recurrent otitis. In addition, the study can also promote the understanding of the development of binaural neural processes in neonates and children with and without hearing disorders and the impact of the history of infection by conductive component in these processes.

\section{Objective}

To characterize and compare monoaural and binaural auditory responses in neonates and children without and with a history of recurrent otitis.

\section{Methods}

The present study is cross-sectional and documentary. It was performed after approval by the research ethics committee of the institution where it was performed in accordance with resolution 466/2012, and its approval number is 56892216.2.0000.5406/2016. All parents and guardians authorized the research and signed a free and informed consent form.

Regarding the sample, 72 subjects participated in the study, 43 boys and 29 girls, aged 1 month to 8 years and 11 months old. The subjects were distributed into two groups, with no history of recurrent otitis (control group [CG]) and with history of recurrent otitis (study group [SG]).

Control group: 37 babies and children without hearing complaints and with no history of hearing disorders, selected by spontaneous demand at the institution. All participants in this group had hearing within normal thresholds, with tonal thresholds, as evidenced by type "A" tympanometry, ${ }^{10}$ puretone audiometry (PTA), or visual reinforcement audiometry (VRA) at $20 \mathrm{dBHL}$ (ANSI) and/or who passed the hearing screening with click-stimulus brainstem auditory evoked potential (BAEP-click) with wave $\mathrm{V}$ at $30 \mathrm{dBHL}^{11}$; in addition to the presence of a response in the otoacoustic emissions 
(OAE) testing. The subjects did not present any neurological, behavioral, or psychological impairments, confirmed by anamnesis with the parents or verified in the medical history anamnesis.

Study group: 35 babies and children with a history of up to three or more episodes of bilateral or unilateral secretory otitis media during childhood, diagnosed and monitored by the institution's otolaryngologist. The history of infection was confirmed by anamnesis with the parents and guardians at the time of assessment and verified in the anamneses and previous audiological exams in the medical history. All participants in this group had hearing within normal thresholds at the time of collection assessment, with tonal thresholds, as evidenced by type A tympanometry, ${ }^{10}$ pure tone audiometry (PTA) or visual reinforcement audiometry (VRA) at $20 \mathrm{dBHL}$ (ANSI) and/or who passed the hearing test with BAEP-click with the presence of wave $\mathrm{V}$ at $30 \mathrm{dBHL}^{11}$; in addition to the presence of a response in the OAE testing. Tympanometry was performed with a $1,000 \mathrm{~Hz}$ probe for neonates and a $226 \mathrm{~Hz}$ one for children over 12 months. ${ }^{12}$ Normal curves were considered the ones with tympanometric peak pressure between -100 to +50 daPa, and for static compliance, from 0.20 to $0.68 \mathrm{ml} .{ }^{10}$ This information was collected in the medical record prior to the collection procedure.

As for the collection procedure, the CAEP was performed using Bio-logic Navigator Pro equipment (Natus Medical Inc., Pleasanton, CA, USA) and recorded using five disposable electrodes positioned at Fpz, the ground electrode, Fz and $\mathrm{Cz}$, these as the active electrodes, in reference to electrodes A1 and A2, which were in the subject's left and right earlobes ( $\mathrm{Fz}$ and $\mathrm{Cz} / \mathrm{A} 1$ and $\mathrm{Fz}$ and $\mathrm{Cz} / \mathrm{A} 2$ ), using the 2 recording channels of the equipment to stimulate the right and left ears and bilaterally. Thus, in all recordings, the same electric field was used for the subsequent BIC calculation.

Impedance was maintained below 5 Kohms. Speech stimulus /ba/-/da/ was used to acquire CAEP. ${ }^{13}$ Stimuli were randomly presented as monoaurals in the $2 \%$ proportion of rare stimuli /da/ out of a total of 100 stimuli /ba/ - oddball paradigm, recorded in a 500 milliseconds window with 1 to $30 \mathrm{~Hz}$ bandpass filtering, 50,000x amplification, alternating polarity and stimulation rate at 1.9 stimuli/second. Natural speech stimuli were fluid $180 \mathrm{~ms}$-duration male voices recorded at $70 \mathrm{dBHL}$, extracted from the second syllable during the emission [dada], in which formants F1, F2, and F3 were obtained in their initial and stable portion. Such stimuli were developed at the linguistics laboratory of the Institute of Language Studies, at Universidade de Campinas, recorded in Praat (Version 4.2.31, Phonetic Sciences, University of Amsterdam), at $48 \mathrm{KHz}$, and then recorded in $\mathrm{CD}$ for wave format insertion and insertion in the Bio-logic Navigator (Natus) equipment software.

The activity of eye movement was indirectly controlled, the electrical activity of the brain was monitored in real time throughout the examination. When the voltage exceeded the baseline, the examination was stopped, and ocular fixation was requested. The examination was only resumed when the cortical activity remained stable.

After examination collection, P1-N1-P2-N2 complex was identified-first waves that appear in sequence and present negative-positive-negative polarity, respectively, according to the patient's chronological age, always performed in the " $\mathrm{Cz}$ " vertex recording of the infrequent stimulus /da/. Such identification was performed by the researcher and afterwards by two judges, experts in the field of electrophysiology with no personal identification of the subjects or information regarding age groups or groups.

The analyses and calculations were developed by the researchers of the institution's research laboratory ${ }^{14}$ using the formula: $\mathrm{BIC}=([\mathrm{D}+\mathrm{E}]-\mathrm{BIN})$. At first, the wave data for all patients were extracted from the software AEP to AESCII (Biologic) and transposed into an Excel spreadsheet (Microsoft Corp., Redmond, WA, USA). The numerical information of the 512 CAEP wave points was compiled and mathematically analyzed for calculating BIC. Such analysis corresponded to the sum of the predetermined waves in an Excel algorithm obtained by the stimulation of the right (D) and left (E) ears followed by the subtraction of the wave obtained by the bilateral stimulation.

In relation to the statistical analysis, 2-way mixed repeated measures analysis of covariance (ANCOVA) tests were performed to analyze the group effect (CG and SG), the condition (right, left, and bilateral stimulation) and the interaction (group versus condition), controlling the effect of age-sex covariates.

Box's M test was used to verify whether the covariance matrices of the observed dependent variables are the same for both groups, and Mauchly's sphericity test was used to test the sphericity hypothesis. In case of sphericity hypothesis rejection, the analyzes were based on the GreenhouseGeisser multivariate test.

The main effect within the group and/or condition was analyzed using the Bonferroni multiple comparison test. The adopted confidence level was $5 \%$. The data were analyzed using IBM SPSS for Windows version 24.0. (IBM Corp. Armonk, NY, USA). The data are presented in the results in tables and graphs.

\section{Results}

The results below present the descriptive and comparative analyses of the monoaural and binaural auditory responses of children from the CG and SG.

As for the CAEP, after statistical analysis, a significant difference was observed between CG and SG groups for all latency values; and for the latencies of the P1, N1, P2, and N2 potentials, the differences between the groups occurred in the three analyzed conditions (RE, LE, and BILAT), which demonstrates the influence of the history of recurrent otitis for CG (-Table $\mathbf{1}$ ).

However, there were no significant differences in relation to the age groups; that is, statistically there is no direct interference of age in the presented results. In this study, age was not a major factor for changes in CAEP in this population. Regarding gender, only for N1 latency significance was observed in males (- Table 1).

For amplitude, no significant differences were observed between groups, conditions, or interaction (-Table $\mathbf{2}$ ). 
Table 1 Descriptive and comparative analysis of cortical auditory evoked potential latency components of the right, left and bilateral ears

\begin{tabular}{|c|c|c|c|c|c|c|c|}
\hline \multirow[b]{3}{*}{ Condition } & \multicolumn{4}{|c|}{ Groups } & \multicolumn{3}{|c|}{ ANCOVA } \\
\hline & \multicolumn{2}{|c|}{$\begin{array}{l}\text { CG }=\text { Normal } \\
(n=37)\end{array}$} & \multicolumn{2}{|c|}{$\begin{array}{l}\text { SG }=\text { Infection } \\
(n=35)\end{array}$} & \multirow{2}{*}{$\begin{array}{l}\text { Group } \\
p\end{array}$} & \multirow{2}{*}{$\begin{array}{l}\text { Condition } \\
p\end{array}$} & \multirow{2}{*}{$\begin{array}{l}\text { Interaction } \\
p\end{array}$} \\
\hline & Mean & SD & Mean & SD & & & \\
\hline LatRE_Cz_P1 & 73.4 & 19.0 & 87.2 & 27.9 & \multirow[t]{3}{*}{$0.001^{*}$} & \multirow[t]{3}{*}{0.689} & \multirow[t]{3}{*}{0.064} \\
\hline LatLE_Cz_P1 & 73.8 & 21.2 & 87.0 & 26.9 & & & \\
\hline LatBILAT_Cz_P1 & 58.8 & 33.3 & 90.9 & 37.6 & & & \\
\hline LatRE_Cz_N1 & 115.1 & 35.3 & 135.3 & 44.6 & \multirow[t]{3}{*}{$0.001^{*}$} & \multirow[t]{3}{*}{0.249} & \multirow[t]{3}{*}{$0.344 \mathrm{~s}$} \\
\hline LatLE_Cz_N1 & 113.0 & 30.6 & 139.9 & 52.3 & & & \\
\hline LatBILAT_Cz_N1 & 92.0 & 51.5 & 132.4 & 51.9 & & & \\
\hline LatRE_Cz_P2 & 166.1 & 41.9 & 193.7 & 44.9 & \multirow[t]{3}{*}{$0.001^{*}$} & \multirow[t]{3}{*}{0.483} & \multirow[t]{3}{*}{0.334} \\
\hline LatLE_Cz_P2 & 175.4 & 37.0 & 206.0 & 46.4 & & & \\
\hline LatBILAT_Cz_P2 & 148.5 & 70.7 & 196.7 & 57.5 & & & \\
\hline LatRE_Cz_N2 & 225.2 & 46.4 & 258.4 & 46.3 & \multirow[t]{3}{*}{$0.001^{*}$} & \multirow[t]{3}{*}{0.406} & \multirow[t]{3}{*}{0.596} \\
\hline LatLE_Cz_N2 & 241.6 & 46.9 & 270.3 & 53.5 & & & \\
\hline LatBILAT_Cz_N2 & 212.2 & 101.8 & 261.6 & 63.8 & & & \\
\hline
\end{tabular}

Abbreviations: ANCOVA, analysis of covariance; BILAT, bilateral; CG, control group; Lat, latency; LE, left ear; RE, right ear; SD, standard deviation; SG, study group.

Note: ${ }^{*} p \leq 0.05$ for significant effect by ANCOVA; $s p \leq 0.05$ for significant effect of sex covariate; equal superscript letters indicate significant differences by Bonferroni multiple comparison test.

Table 2 Descriptive and comparative analysis of cortical auditory evoked potential amplitude components of the right, left and bilateral ears and group effect and condition

\begin{tabular}{|c|c|c|c|c|c|c|c|}
\hline \multirow[b]{3}{*}{ Condition } & \multicolumn{4}{|l|}{ Groups } & \multicolumn{3}{|c|}{ ANCOVA } \\
\hline & \multicolumn{2}{|c|}{ CG $(n=37)$} & \multicolumn{2}{|c|}{ SG $(n=35)$} & \multirow{2}{*}{$\frac{\text { Group }}{p}$} & \multirow{2}{*}{$\begin{array}{l}\text { Condition } \\
p\end{array}$} & \multirow{2}{*}{$\begin{array}{l}\text { Interaction } \\
p\end{array}$} \\
\hline & Mean & SD & Mean & SD & & & \\
\hline AmplRE_Cz_P1 & 4.04 & 4.12 & 3.66 & 3.31 & \multirow[t]{3}{*}{0.051} & \multirow[t]{3}{*}{0.268} & \multirow[t]{3}{*}{0.143} \\
\hline AmplLE_Cz_P1 & 2.08 & 2.33 & 4.38 & 4.12 & & & \\
\hline AmplBILAT_Cz_P1 & 2.66 & 4.07 & 4.96 & 5.23 & & & \\
\hline AmplRE_Cz_N1 & -2.34 & 3.31 & -2.22 & 2.84 & \multirow[t]{3}{*}{0.839} & \multirow[t]{3}{*}{0.942} & \multirow[t]{3}{*}{0.733} \\
\hline AmplLE_Cz_N1 & -2.44 & 2.59 & -2.87 & 2.50 & & & \\
\hline AmplBILAT_Cz_N1 & -1.56 & 3.21 & -1.08 & 3.96 & & & \\
\hline AmplRE_Cz_P2 & 3.40 & 3.04 & 2.96 & 2.87 & \multirow[t]{3}{*}{0.770} & \multirow[t]{3}{*}{0.373} & \multirow[t]{3}{*}{0.536} \\
\hline AmplLE_Cz_P2 & 2.89 & 3.05 & 2.66 & 4.39 & & & \\
\hline AmplBILAT_Cz_P2 & 3.45 & 5.02 & 4.29 & 3.69 & & & \\
\hline AmplRE_Cz_N2 & -5.27 & 3.58 & -5.48 & 3.85 & \multirow[t]{3}{*}{0.306} & \multirow[t]{3}{*}{0.633} & \multirow[t]{3}{*}{0.595} \\
\hline AmplLE_Cz_N2 & -4.34 & 3.50 & -5.99 & 3.78 & & & \\
\hline AmplBILAT_Cz_N2 & -5.41 & 5.66 & -6.21 & 4.87 & & & \\
\hline
\end{tabular}

Abbreviations: Ampl, amplitude; ANCOVA, analysis of covariance; BILAT, bilateral; CG, control group; LE, left ear; RE, right ear; SD, standard deviation; SG, study group.

Note: ${ }^{*} p \leq 0.05$ for significant effect by ANCOVA; $s p \leq 0.05$ for significant effect of sex covariate; equal superscript letters indicate significant differences by Bonferroni multiple comparison test.

There was no significance for any value or presented condition; however, P1 is observed as the only one approaching the significant value of $p \leq 0.05$ in the comparison between groups.

It is noteworthy that the amplitude value means of P1 for RE and BILAT is higher in the SG, which can demonstrate a functional difference between ears in this group.
Next, - Fig. 1 shows the recording obtained from the grand average of BIC component, calculated from monoaural and binaural recordings in the two groups (CG and SG) will be presented. The geometric shapes represent the appearance of two positive peaks and two negative peaks.

Regarding BIC, there is a difference between the groups in the characteristics of the peaks obtained from the calculation 


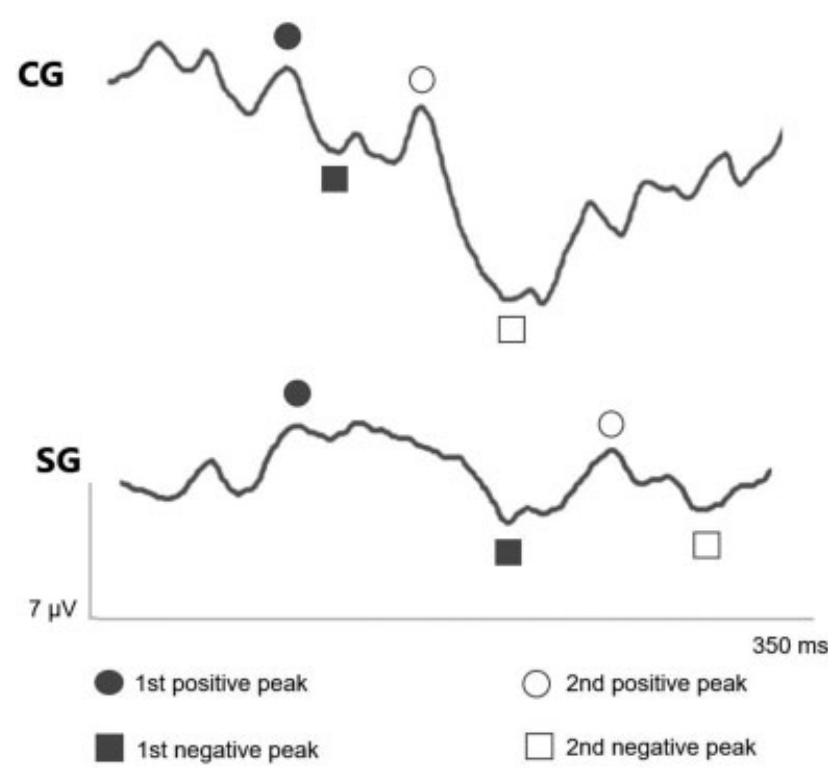

Fig. 1 Binaural interaction component (BIC) Cortical auditory evoked potential - Control group and study group.

of the binaural integration component, with increased latency and reduced amplitudes for the subjects in the SG.

\section{Discussion}

The P1-N1-P2-N2 wave complex of the CAEP was found in all children participating in the study, even those up to 1 year old. According to the literature in the area, P1 and N1 components tend to be better visualized in children from 5 or 8 years old. ${ }^{15}$

The P1 component is a biomarker of auditory maturation $^{16,17}$ over the years and greater exposure to different auditory stimuli; it tends to decrease in relation to its latency and amplitude, similarly to other components such as N1, whose value also decreases over time, reflecting development. ${ }^{15,18}$ And P2, which, with greater auditory stimulation and auditory training, tends to have decreased latency and amplitude over time, demonstrating the effect of auditory and language learning. ${ }^{19}$

In the present study, statistically significant difference was found for all latency values between groups; however, without direct interference from age. Regarding the analyzed conditions, significant difference was also found for all (LE, RE, and BILAT), and for the latencies of P1, N1, P2, and N2 components, the differences between the groups occurred in the three analyzed conditions: LE, RE, and BILAT.

The results suggest that there are differences in the electrical response of the entire central auditory pathway in the analyzed population and age group, represented by the statistical difference between the components of the wave complex. Regardless of the side affected by the infection and the etiology, otitis primarily affects the peripheral auditory pathway, ${ }^{4}$ and the CANS can undergo changes, in the long term, in the presence of recurrent otitis. ${ }^{9}$ Although some isolated values of individuals in the SG are within the normal standards for CAEP, ${ }^{7}$ when comparing with children with no history of alterations, there is a statistical difference between them, which may reflect differences in binaural development, since for bilateral stimulation, statistical differences occurred for all components of the wave complex.

There was no statistical difference in amplitude between the groups (-Table 2); however, considering the mean values, in general, the SG obtained higher numerical values, demonstrating greater difficulty in capturing the responses to CAEP verbal stimulus, which, in addition, may indicate delayed auditory maturation in these individuals. What is expected is that over the years and with greater exposure to the sound world, the auditory pathway matures ${ }^{21}$ and acquires the ability to respond and perform auditory detection and discrimination tasks automatically and faster, which would be more compatible with a decrease in the amplitude values of the wave components; this could be observed in the CG, but not in the SG.

In the results found in the present study, the higher numerical values of the SG compared with the CG may indicate that in the presence of a history of recurrent otitis, maturation and learning of skills tend to be delayed. The grand averages presented by graphs 2 to 4 help in this reflection, as it is possible to observe how much the history of recurrent otitis interferes in the morphology of the wave components, especially in the recordings of the grand bilateral average.

Regarding the analysis of binaurality and the binaural interaction component (BIC), the observed morphology of the grand average of the recordings was flattened, of low amplitude, worse in the SG group, especially considering that the binaural response goes through several structures of the central and peripheral auditory pathways, including the contralateral ear. ${ }^{2}$ Thus, regardless of the side affected by the middle ear infection, the binaural pathway will have more difficulty in processing the information to be captured by bilateral CAEP.

As the central structures responsible for binaurality, such as the lower and upper colliculi, are the main structures involved in hearing, ${ }^{2}$ we can assume that any change in the reception and processing of auditory information by these structures will influence the acquisition of binaural hearing skills.

In addition, the history of recurrent otitis, which leads to sensory deprivation in children in the development phase, produces losses in the long term, in the perception of the auditory cues of interaural differences in time and level to be understood and developed. ${ }^{3}$

Binaural interaction component in neonates can vary greatly as to the appearance of the complex waves, demonstrating immaturity in the binaural interaction of these subjects, especially with faster stimuli, such as BAEP with click. ${ }^{21}$ A previous study that analyzed BIC when CAEP was recorded with high-intensity click stimulus showed shorter latencies in children with external ear changes than in adults. ${ }^{22}$ However, no studies relating BIC of CAEP in children with middle ear changes were found.

Studies discussing changes in the middle ear and the findings regarding binaurality were performed with other auditory evoked potentials, such as BAEP. These investigations found that when researching these potentials in children with otitis media, their components may have increased 
latency, latency asymmetries between the RE LE and difficulties in binaural interaction. ${ }^{9}$ The BIC results of the present study prove the latency differences between children in the CG and SG also in relation to the morphology of the BIC waves.

Thus, the hypotheses of the study were confirmed; there were differences in the CAEP results in relation to the latency values of all components of the wave, in the three analyzed conditions (LE, RE, and BILAT), between the groups. Furthermore, the hypothesis that sensory deprivation resulting from a history of conductive component due to middle ear infections was confirmed; it may affect the maturation and development of the binaural interaction component between the central auditory pathways.

Such findings reinforce the need for hearing health promotion and prevention. Parents and family members need to be better informed about the consequences of recurrent otitis, the risk of sensory deprivation and the need for audiological monitoring in these cases.

For the audiology scientific community, it is important to reflect on the need to assess binaurality in cases of children with a history of recurrent otitis. More investments are needed for further research to assess binaurality in children with different types of hearing conditions, ages, and clinical conditions.

\section{Conclusion}

There are differences between CAEP responses in relation to the latency values of the wave components in all the analyzed conditions, LE, RE, and BILAT. There are maturational differences in babies and children with and without history of recurrent otitis, especially for bilateral stimulation for all CAEP wave components, which was also confirmed by BIC wave analysis.

\section{Funding Source}

Coordination for the Improvement of Higher Education Personnel (CAPES, in the Portuguese acronym).

\section{Conflict of Interests}

The authors have no conflict of interests to declare.

\section{References}

1 Isaac ML, Oliveira JAA. Tratado de implante coclear e próteses auditivas implantáveis, 2nd ed. Rio de Janeiro: Thieme; 2014

2 Goffi-Gomez MVS. Tratado de implante coclear e próteses auditivas implantáveis,2nd ed. Rio de Janeiro: Thieme; 2014

3 Avan P, Giraudet F, Büki B. Importance of binaural hearing. Audiol Neurotol 2015;20(01, Suppl 1):3-6
4 Cardoso A. Reflexões sobre o desenvolvimento auditivo. Verba Volant 2013;4(01):104-116

5 Borges LR. Audiological and behavior findings in children underwent a bilateral myringoplasty - a comparative study. Rev CEFAC 2016;18(04):881-888

6 Spineli EA. Maloclusões e disfunção da tuba auditiva em crianças: uma revisão integrativa. Rev CEFAC 2016;18(04):960-964

7 Kraus N, Nicol T. Aggregate neural responses to speech sounds in the central auditory system. Speech Commun 2003;41(01):35-47

8 McPherson DL, Starr A. Binaural interaction in auditory evoked potentials: brainstem, middle- and long-latency components. Hear Res 1993;66(01):91-98

9 Roberts J, Hunter L, Gravel J, et al. Otitis media, hearing loss, and language learning: controversies and current research. J Dev Behav Pediatr 2004;25(02):110-122

10 Jerger J. Clinical experience with impedance audiometry. Arch Otolaryngol 1970;92(04):311-324

11 Souza LCA, Piza MRT, Alvarenga KF, Cóser PL. Eletrofisiologia da audição e emissões otoacústicas, 3nd ed. São Paulo: Book Toy; 2016

12 Teixeira BN, Sleifer P, Pauletti LF, Krimberg CFD. Study of acoustic immittance measures with probe tone of 226 and $1000 \mathrm{~Hz}$ in neonates. ACR 2013;18(02):126-132

13 Banhara MR, Costa Filho OA. Potenciais auditivos de longa latência: N1, P2, N2, P300, evocados por estímulo de fala em usuários de implante coclear [dissertação]. Bauru: Universidade de São Paulo, Faculdade de Odontologia de Bauru; 2007

14 Oliveira LS, Frizzo ACF. Componente de interação binaural do potencial evocado auditivo cortical em crianças com histórico de otite de repetição [dissertação]. Marília: Universidade Estadual Paulista, Faculdade de Filosofia e Ciências; 2018:62

15 Sharma A, Kraus N, McGee TJ, et al. Developmental changes in P1 and $\mathrm{N} 1$ central auditory responses elicited by consonant-vowel syllables. Electroencephalography and Clinical Neurophysiology -. Evok Pot 1997;104(06):540-545

16 Alvarenga KdeF, Vicente LC, Lopes RC, et al. The influence of speech stimuli contrast in cortical auditory evoked potentials. Rev Bras Otorrinolaringol (Engl Ed) 2013;79(03):336-341

17 Silva LAF, Couto MIV, Matas CG, Carvalho ACM. Long latency auditory evoked potentials in children with cochlear implants: systematic review. CoDAS 2013;25(06):595-600

18 Sussman E, Steinschneider M, Gumenyuk V, Grushko J, Lawson K. The maturation of human evoked brain potentials to sounds presented at different stimulus rates. Hear Res 2008;236(12):61-79

19 Tremblay KL, Ross B, Inoue K, McClannahan K, Collet G. Is the auditory evoked $\mathrm{P} 2$ response a biomarker of learning? Front Syst Neurosci 2014;8(28):28

20 Cone-Wesson B, Ma E, Fowler CG. Effect of stimulus level and frequency on ABR and MLR binaural interaction in human neonates. Hear Res 1997;106(1-2):163-178

21 Ponton CW, Eggermont JJ, Kwong B, Don M. Maturation of human central auditory system activity: evidence from multi-channel evoked potentials. Clin Neurophysiol 2000;111(02):220-236

22 Sheykholeslami K, Mohammad HK, Sébastein S, Kaga K. Binaural interaction of bone-conducted auditory brainstem responses in children with congenital atresia of the external auditory canal. Int J Pediatr Otorhinolaryngol 2003;67(10):1083-1090 